\title{
Conserved functional domains of the RNA polymerase III general transcription factor BRF
}

\author{
Bernard Khoo, Brigid Brophy, and Stephen P. Jackson ${ }^{1}$ \\ Wellcome/CRC Institute and Department of Zoology, Cambridge University, Cambridge CB2 1QR, UK
}

In Saccharomyces cerevisiae, two components of the RNA polymerase III (Pol III) general transcription factor TFIIIB are the TATA-binding protein (TBP) and the B-related factor (BRF), so called because its amino-terminal half is homologous to the Pol II transcription factor IIB (TFIIB). We have cloned BRF genes from the yeasts Kluyveromyces lactis and Candida albicans. Despite the large evolutionary distance between these species and $S$. cerevisiae, the BRF proteins are conserved highly. Although the homology is most pronounced in the amino-terminal half, conserved regions also exist in the carboxy-terminal half that is unique to BRF. By assaying for interactions between BRF and other Pol III transcription factors, we show that it is able to bind to the 135-kD subunit of TFIIIC and also to TBP. Surprisingly, in addition to binding the TFIIB-homologous amino-terminal portion of BRF, TBP also interacts strongly with the carboxy-terminal half. Deleting two conserved regions in the BRF carboxy-terminal region abrogates this interaction. Furthermore, TBP mutations that selectively inhibit Pol III transcription in vivo impair interactions between TBP and the BRF carboxy-terminal domain. Finally, we demonstrate that BRF but not TFIIB binds the Pol III subunit C34 and we define a region of $\mathrm{C} 34$ necessary for this interaction. These observations provide insights into the roles performed by BRF in Pol III transcription complex assembly.

[Key Words: Transcription; general transcription factor; RNA polymerase III; TFIIIB; BRF; TBP]

Received June 16, 1994; revised version accepted September 30, 1994.

In eukaryotic cell nuclei there are three DNA-dependent RNA polymerases. Each relies on a set of basal transcription factors to direct it to transcriptional promoters and to transduce signals from regulatory proteins. Although the basal factors employed by a particular polymerase are distinct from those employed by the others, one factor, the TATA-binding protein (TBP), is a component of all three systems (for reviews, see White and Jackson 1992b; Hernandez 1993; Rigby 1993; Struhl 1994). TBP is able to participate in these disparate roles by associating with three distinct sets of TBP-associated factors (TAFs) to form the complexes SLl, transcription factor IID (TFIID), and TFIIIB, which are dedicated to Pol I, Pol II, and Pol III, respectively (Dynlacht et al. 1991; Buratowski and Zhou 1992; Colbert and Hahn 1992; Comai et al. 1992; Kassavetis et al. 1992; Lobo et al. 1992; Pugh and Tjian 1992; Simmen et al. 1992; Taggart et al. 1992; White and Jackson 1992a; Chiang et al. 1993; Eberhard et al. 1993; Huet and Sentenac 1993). The most important Pol III basal transcription factor is TFIIIB, as this is the only factor utilized by all Pol III templates. TFIIIB comprises TBP and polypeptides of $\sim 70$ and $90 \mathrm{kD}$ (Kassavetis et al. 1992). Although TFIIIB contains TBP as one of its sub-

\footnotetext{
${ }^{1}$ Corresponding author.
}

units, it does not recognize Pol III promoters primarily by sequence-specific DNA recognition. Instead, it is recruited to class III genes by protein-protein interactions with other Pol III basal factors, such as TFIIIC, which are bound to specific promoter sequences /Geiduschek and Tocchini-Valentini 1988; Kassavetis et al. 1992; Willis 1993; White 1994). In the process of associating with class III templates, however, TFIIIB interacts intimately with DNA upstream from the transcription initiation site (Kassavetis et al. 1989, 1991; Bartholomew et al. 1991) and, in yeast at least, is sufficient to direct several rounds of transcription initiation, even after TFIIIC has been removed by heparin treatment (Kassavetis et al. 1990). It has thus been concluded that TFIIIB mediates the specific recruitment of Pol III to class III templates.

Recently, genetic screens in Saccharomyces cerevisiae have identified a gene that, when overexpressed, serves as an allele-specific suppressor of point mutations in TBP (Buratowski and Zhou 1992; Colbert and Hahn 1992). This same gene was also isolated in a separate screen selecting for suppressors of a mutation in a tRNA gene promoter (Lopez-de-Leon et al. 1992). Biochemical and genetic analyses have established that the protein product of this gene, variously named BRF, TDS4, or PCF4 and henceforth referred to as BRF, is the $70-\mathrm{kD}$ component of TFIIIB and is essential for yeast viability 
(Buratowski and Zhou 1992; Colbert and Hahn 1992; Kassavetis et al. 1992; Lopez-de-Leon et al. 1992). Interestingly, sequence analysis reveals that the amino-terminal half of BRF is related to the Pol II basal factor TFIIB. TFIIB interacts with both TBP and Pol II and, by serving as a molecular bridge between these proteins, recruits Pol II to the promoter (Tschochner et al. 1992; Wampler and Kadonaga 1992; Barberis et al. 1993; Ha et al. 1993; Hisatake et al. 1993; Malik et al. 1993; Yamashita et al. 1993). TFIIB has also been implicated in selecting the Pol II transcription start site and in responding to regulatory factors (Lin et al. 1991; Lin and Green 1991; Pinto et al. 1992; Roberts et al. 1993). The homology between BRF and TFIIB implies that these factors play parallel roles in the Pol III and Pol II transcriptional machineries. More specifically, it suggests that BRF interacts directly with TBP and Pol III and that BRF may be involved in transducing regulatory signals to the Pol III transcriptional apparatus.

A powerful approach in defining the functional domains of a protein is to determine the regions most conserved in sequence throughout evolution. For example, the comparison of TFIIB polypeptides from diverse eukaryotes has identified conserved regions that are essential for TFIIB activity (Wampler and Kadonaga 1992; Buratowski and Zhou 1993; Na and Hampsey 1993). Because BRF has so far only been cloned from S. cerevisiae, its conserved domains have not been defined. Here, we describe the isolation and analysis of BRF genes from two yeast species that are evolutionarily highly diverged from S. cerevisiae. By comparing the sequences of these three homologs, we identify regions that are strongly conserved and likely to be crucial for BRF function. Furthermore, through in vitro protein-protein binding assays, we investigate interactions between BRF and other Pol III transcription factors and define the regions of the proteins necessary for these interactions. These approaches lead us to a better understanding of the roles played by BRF in Pol III transcription complex assembly and suggest a mechanistic basis for polymerase specificity.

\section{Results}

Cloning the BRF gene of Kluyveromyces lactis

Although basal transcription factors are generally conserved well throughout evolution in terms of overall structure and mechanism of action, the level of amino acid sequence homology varies considerably from one factor to another. For example, whereas the carboxy-terminal 180 amino acid residues of TBP are $>80 \%$ identical between S. cerevisiae and human, TFIIB is only $35 \%$ identical between these species (Pinto et al. 1992; Hernandez 1993). To determine how well BRF is conserved and, thus, define functionally important regions, we attempted to clone its homologs from other species.

Initially, we focused on the yeast Kluyveromyces lactis, which is estimated to have diverged from S. cerevisiae $\sim 10^{8}$ years ago (Barns et al. 1991; Van de Peer et al.
1992). The strategy was to employ low-stringency nucleic acid hybridization, using the $S$. cerevisiae BRF gene as the probe. Southern blotting experiments revealed a single hybridizing locus in the $K$. lactis genome (data not shown). Using conditions optimized for these Southern blots, a $K$. lactis genomic DNA library screen yielded several hybridizing clones. Sequencing one of these revealed a single long open reading frame capable of encoding a protein of 556 amino acid residues with predicted mass of 62,243 daltons. Sequence analysis shows that this protein has strong homology to $S$. cerevisiae BRF (Fig. 1A). In particular, the region encompassing the amino-terminal 270 amino acid residues is $\sim 75 \%$ identical. Although still extensive, the homology in the carboxy-terminal half of these molecules is less pronounced (overall $51 \%$ identity) and contains several short gaps. The strong homology between $S$. cerevisiae and $K$. lactis BRF proteins is reflected in very similar hydropathy profiles (Fig. 1B) and secondary structure predictions (data not shown).

\section{Cloning the BRF gene from Candida albicans}

Next, we attempted to isolate a BRF homolog gene from the more highly diverged yeast Candida albicans. Because preliminary low-stringency Southern blotting experiments were ambiguous, we turned to a polymerase chain reaction (PCR) strategy that has been used successfully to isolate TBP clones from evolutionarily diverse species (Kao et al. 1990; Rowlands et al. 1994). From the sequence comparison of $S$. cerevisiae and $K$. lactis BRFs (Fig. 1), we identified several regions of extended amino acid identity. As described in the legend to Figure 2A, degenerate oligonucleotide primers corresponding to several of these motifs were generated and used in lowstringency PCR reactions with the genomic DNA of $C$. albicans. Through this route, we isolated a gene encoding the entire putative $C$. albicans BRF homolog. Sequencing revealed a single long open reading frame encoding a protein of 553 amino acid residues with a predicted mass of 61,834 daltons. A three-way comparison of the $S$. cerevisiae, $K$. lactis, and $C$. albicans BRF homologs is shown in Figure 2A. Conservation is most pronounced in the amino-terminal TFIIB homologous region, especially in the area comprising the direct repeats. However, three blocks of strong homology are also identifiable in the BRF-specific carboxyl terminus (Fig. 2A,B; also see Discussion). Particularly in the amino-terminal half of these molecules, sequence homologies are reflected in very similar hydropathy profiles and secondary structure predictions (data not shown).

\section{C. albicans $B R F$ complements an S. cerevisiae $B R F$ mutant}

The fact that the $C$. albicans protein sequence described above has extensive homology throughout its length with $S$. cerevisiae BRF suggests strongly that it corresponds to a functional BRF homolog. To test this, we determined whether the growth defect of an $S$. cerevisiae 
A

KLBRF MASTLLVSSRKCKNCGSTDFVRDISNTTINELICKVCGLVTEENSIVSELAFGEASNGAAV SCBRF MPV--- - CKNCHGTEFERDLSNANNDLVCKACGVVSEDNPIVSEVTFGETSAGAAV

KLBRF IQGAFVSANQAHPTFMSHSGQNALMSRETTLNNARRKLKAVSYALNIPEYVTDAAFOWYR

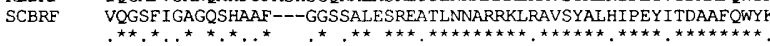

KLBRF LALSNNFVQGRKSQNVIAACLY IACRKERTHHMLIDFSSRLQVSVYSIGATFLKLAKKLQ LALANNFVQGRRSQNVIASCLYYACRKEKTHHMLIDF SSRLQVSVYSIGATF LKMVKKLL SCBRF ITELPLADPSLFIQHFAEKLDIADKKIIVVKRDAVKLAQRMSKDWMFEGRRPAGIAGACII $\underset{.}{\longrightarrow}$

KLBRF LACRMNNLRRTHSEIVAIS HVAEETLQQRLNEFKNTTSSAKLSVKEFRDDETEVNEGERSA $S C B R F$ LACRMNNLRRTHTEIVAVSHVAEETLQQRLNEEFKNTKAAKLSVQKFRENDVE

KLBRF ESKPPSFDKNRLKEKKIKDSLDTKEMLETSEEAVSRNP ILTQVLGGAQELSSKEVLYYLKK EARPPSFVKNRKKERKIKDSLDKEEMFQTSEEALNKNR ILTQVLGEQELSSKEVLFYLKQ $\begin{array}{ll}\text { KLBRF } & \text { LSERRKAEFSHIKATHGIDGEDLHKTEKD---KKRSLDE---- } \\ \text { SCBRF } & \text { FSERRARVVERIKATNGIDGENIYHEGSENETRKRKLSEVSIONEHVEGEDKETEGTEEK }\end{array}$

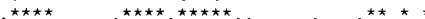

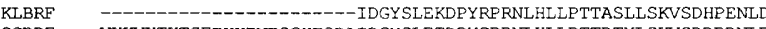
VKKVKTKTSEEKKENESGHFQDAIDGYSLETDPYCPRNLHLLPTTDTYLSKVSDDPDNLE

KLBRF DVDDAELDSHLLDEEASKLKERIWIDINGDYLIEQESKRLKQEADLASGNTSLRKKRSKR SCBRF DVDDEELNAHLLNEEASKL KERIWIGLNADFLLEQESKRLKOEADIATGNTSVKKKRTRR

KLBRE TNRNQSSASIVKVQVD---GL--------PLDVSVDDAD-AVDVVAAGGVKNLLQKTTFS SCBRF RNNTRSDEPTKTVDAAAAIGLMSDLQDKSGLHAALKAAEESGDFTTADSVKNMLQKASFS KLBRF KKINYDAINGLFGQK KKINYDAIDGLF--R
B

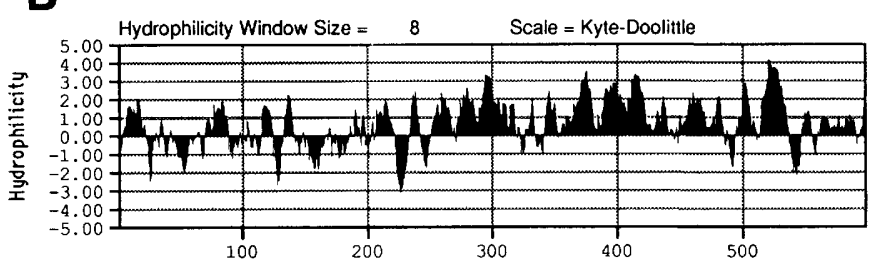

S. cerevisiae BRF

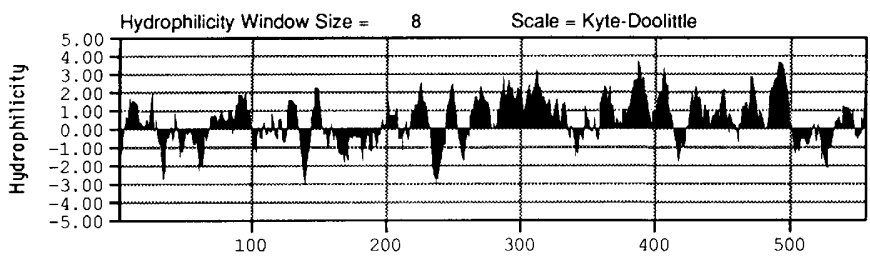

K. lactis BRF

Figure 1. (A) Cloning and sequence analysis of the $K$. lactis BRF gene. A $K$. lactis genomic DNA plasmid library was probed with a radiolabeled DNA fragment encompassing the amino-terminal region of $S$. cerevisiae $\mathrm{BRF}$ that is related to TFIIB. Low-stringency hybridization and washing protocols were employed (final wash of $2 \times \mathrm{SST}$ at $50^{\circ} \mathrm{C}$ ). A hybridizing clone was thus identified and sequenced. The single long open reading frame in the $K$. lactis DNA insert is presented in a comparison with the $S$. cerevisiae BRF protein sequence. Identities and similarities are indicated by asterisks and dots, respectively. The putative zinc finger and direct repeats in the TFIIB-homologous domain are indicated by the bracket and arrows, respectively. $(B)$ Hydropathy plots of $S$. cerevisiae and $K$. lactis BRF homologs were derived by the method of Kyte and Doolittle (1982).

plex (Bartholomew et al. 1991). As an approach to analyze potential interactions between BRF and $\tau 135$, we performed in vitro protein-protein binding "pulldown" assays. To do this, a fusion of glutathione $S$-transferase (GST) with full-length $S$. cerevisiae BRF was synthesized in Escherichia coli and purified by adsorption to glutathione-agarose beads. The immobilized proteins were then incubated with $\left[{ }^{35} S\right]$ methionine-labeled derivatives of $S$. cerevisiae $\tau 135$ that had been generated by in vitro transcription and translation. After washing the beads extensively, the quantity of protein bound was assessed by SDS-PAGE and fluorography. As can be seen from Figure 3, the amino-terminal, middle, and carboxy-terminal thirds of $\tau 135$ (residues 1-363, 364-714, and 7151025 , respectively) all bind to the GST-BRF fusion. In contrast, little or no binding is observed to GST protein alone or to several control fusions (data not shown). These studies indicate that multiple regions of $\tau 135 \mathrm{can}$ mediate specific contacts with BRF and suggest that these interactions may play an important role in Pol III transcription complex assembly. Interestingly, $\tau 135$ contains 11 copies of the tetratricopeptide repeat (TPR) motif (Marck et al. 1993), and each of the three portions of this protein that we synthesized contains several of these motifs. As TPR motifs have been suggested to mediate protein-protein interactions (Goebl and Yanagida 
Figure 2. $(A)$ Sequence comparison of $C$. albicans, $K$. lactis, and $S$. cerevisiae BRFs (CABRF, KLBRF, and SCBRF, respectively). For cloning the $C$. albicans BRF gene, lowstringency PCR reactions were performed as described in Materials and methods, using C. albicans genomic DNA and primer pairs corresponding to conserved sequences in $S$. cerevisiae and $K$. lactis BRFs. In one case /primer pair $153 / 156$; corresponding to amino acid sequences THHMLID and QQLTEEAVI, a product was generated of the anticipated size. This, in turn, was radiolabeled and used to isolate a BRF homolog gene from a $C$. albicans genomic DNA library. The translation product of the C. albicans BRF homolog gene is presented in a comparison with the $S$. cerevisiae and $K$. lactis BRF protein sequences. Identities and similarities are indicated by asterisks and dots, respectively. The TFIIBhomologous domain is boxed; within this domain, the putative zinc finger region is indicated by a bracket, the imperfect direct repeats by arrows, and the region that coincides with a predicted basic amphipathic helix of TFIIB by a broken underline. The three regions of strong conservation in the carboxyl terminus (denoted I, II, and III) are underlined. $(B)$ Schematic representation of the BRF sequence conservations shown in $A$. Percentages refer to identities between $K$. lactis or C. albicans proteins with S. cerevisiae BRF.
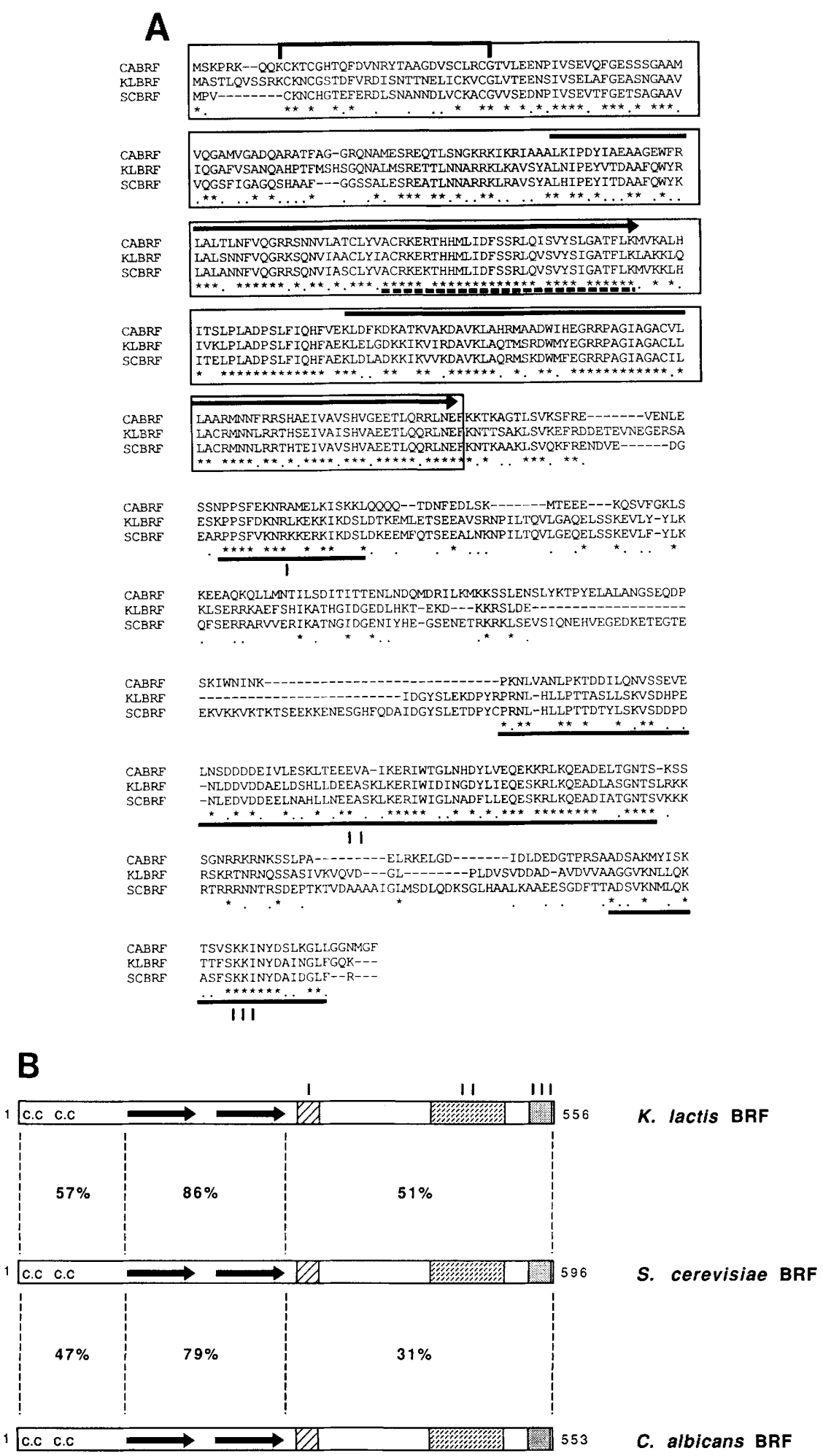

1991), it is possible that these regions direct interactions between $\tau 135$ and BRF.

Two regions of BRF interact with TBP

The overexpression of BRF has been shown previously to suppress the growth defects of $S$. cerevisiae strains containing point mutations in TBP that impair transcription by Pol III (Buratowski and Zhou 1992; Colbert and Hahn 1992). Although this suppression could be mediated by mechanisms that do not involve direct interactions between BRF and TBP, the most likely interpretation is 

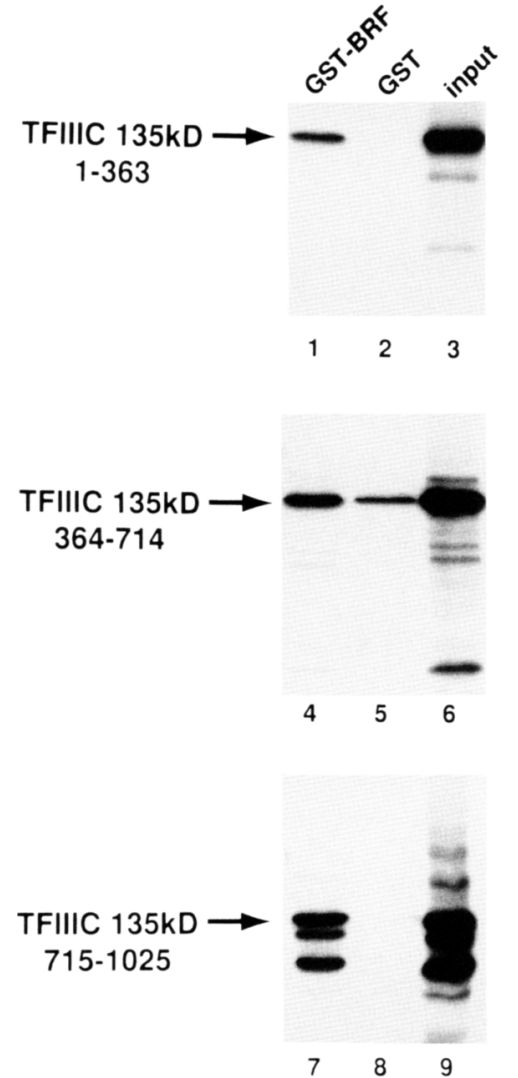

Figure 3. BRF binds to the $135-\mathrm{kD}$ subunit of TFIIIC $(\tau 135)$. Glutathione beads bearing GST/full-length BRF (lanes 1,4,7) or GST alone (lanes 2,5,8) were used in interaction assays as described in Materials and methods with the following ${ }^{35} \mathrm{~S}$-labeled in vitro-translated derivatives of $\tau 135$ : amino acid residues 1-363 (lanes 1-3), 364-714 (lanes 4-6), or 715-1025 (lanes 7-9). Bound proteins were detected by SDS-PAGE followed by autoradiography. To assess the efficiency of protein-protein interactions, the input amount of $\tau 135$ derivatives are included in lanes 3,6 , and 9 and the positions of these proteins on the gel are indicated by arrows. The percentages of the input protein bound were $54 \%, 0 \%, 46 \%, 18 \%, 62 \%$, and $0 \%$ for lanes $1,2,4,5,7$, and 8 , respectively.

that BRF binds to TBP and that BRF overexpression stabilizes TBP in its active conformation. To see whether BRF can bind TBP, we again performed in vitro proteinprotein interaction assays employing GST-BRF fusions. These experiments revealed that $S$. cerevisiae TBP binds to the GST fusion bearing full-length BRF (Fig. 4A, lane 1), whereas no significant binding is observed to GST alone (lane 2) or to several control fusions (data not shown). These results provide strong support for the existence of interactions between BRF and TBP in the Pol III transcription complex.

To elucidate which regions of BRF mediate the interaction with TBP, we tested TBP binding to GST fusions bearing either the amino-terminal TFIIB homologous half of BRF or the carboxy-terminal half that has no counterpart in TFIIB (Fig. 4B). Perhaps surprisingly, in addition to detecting interactions between $S$. cerevisiae TBP and the BRF amino-terminal region (lane 1), strong TBP binding was obtained with the carboxy-terminal BRF domain (lane 4). To further dissect the amino-terminal half of BRF, we synthesized both the putative zinc finger region and the direct repeat region separately as GST fusions and then tested them for TBP binding. This revealed that TBP binds the repeat domain, but only very weakly to the zinc finger region (Fig. 4B, lanes 3 and 2, respectively). Similar results were also obtained using TBP that had been expressed in bacteria, indicating that the interactions between BRF and TBP are likely to be direct rather than being mediated by other proteins in the in vitro transcription/translation system /data not shown). We therefore conclude that two distinct regions of BRF bind TBP: One maps to the amino-terminal direct repeat domain; the other to the carboxy-terminal half of the molecule. The specificity of these interactions is indicated by the fact that other derivatives of BRF, such as 1-89 and 263-431, do not bind TBP detectably despite the fact that these proteins are expressed at high levels in soluble form (Fig. 4C).

Recent studies have identified mutations in $S$. cerevisiae TBP that inhibit Pol III transcription in vivo but do not affect transcription by Pol II (Cormack and Struhl 1993; Kim and Roeder 1994). These mutations map to a solvent-exposed region of TBP that defines a groove across the top of the protein (Kim et al. 1993). It has therefore been suggested that this region specifies a surface of TBP that mediates important interactions with the Pol III transcriptional apparatus. Interestingly, overexpression of BRF in strains bearing temperature-sensitive Pol III-specific TBP mutations results in a partial reversal of the growth-defect phenotype (Cormack and Struhl 1993). We therefore tested whether these mutations affected interactions between TBP and BRF. To do this, three different Pol III-specific TBP mutants were synthesized and compared with wild-type $S$. cerevisiae TBP for binding to various BRF derivatives. Strikingly, compared with wild-type TBP (Fig. 5A), the TBP mutant F155S (Fig. 5B) is impaired drastically in its ability to interact with the carboxy-terminal portion of BRF (Fig. 5, $A$ and $B$, cf. lanes 3 ). In contrast, it can still bind effectively to the amino-terminal region of BRF and fulllength BRF protein (Fig. 5B, lanes 1,2) and actually displays slightly increased binding to these derivatives. Similar results are also obtained with the other two Pol III-specific TBP mutants tested, K138L (Fig. 5C) and $\mathrm{R} 137 \mathrm{~W}$ (data not shown). These data show that the three Pol III-specific TBP mutations tested all specifically impair the binding of TBP to the carboxy-terminal region of BRF and suggest that this protein interaction defect may be responsible for reduced Pol III transcription in strains bearing these mutations.

If the binding of TBP to the carboxy-terminal half of BRF is of physiological significance, one might expect that the regions of BRF mediating this interaction would be conserved throughout evolution. Potential candidates for such regions are the three blocks of homology identified in Figure 2. We found that deletion of the carboxy- 


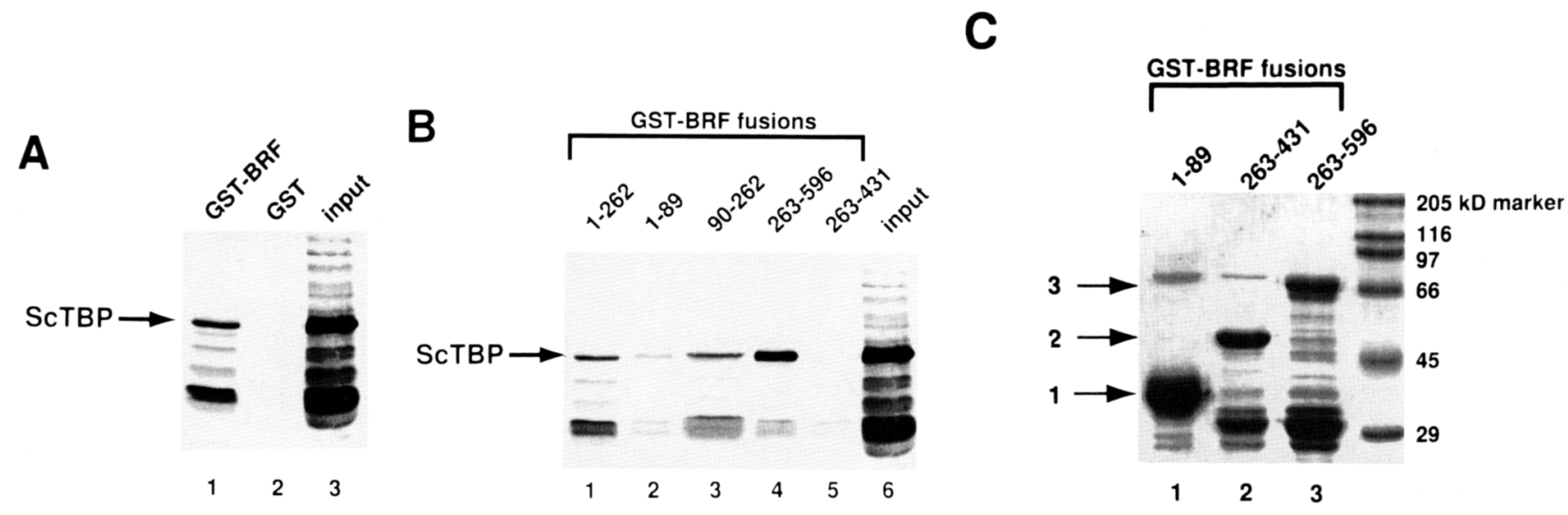

Figure 4. Interactions between BRF and TBP. (A) BRF binds TBP. Glutathione beads bearing GST/full-length BRF (lane 1) or GST alone (lane 2) were tested for binding of ${ }^{35}$ S-labeled in vitro-translated wild-type $S$. cerevisiae TBP as described in Materials and methods. The amount of input protein used in these experiments is presented in lane 3 to allow an assessment of binding efficiency. Percentage of input protein bound in lanes 1 and 2 was $18 \%$ and $0 \%$, respectively. $(B)$ TBP binds to two regions of BRF. The binding of wild-type $S$. cerevisiae TBP was assessed using GST fusions to BRF residues 1-262 (zinc finger together with direct repeat region; lane 1), BRF residues 1-89 (zinc finger region alone; lane 2), BRF residues 90-262 (direct repeat region alone; lane 3), BRF residues 263-596 (carboxy-terminal non-TFIIB-homologous domain; lane 4), or BRF residues 263-431 (carboxy-terminal domain lacking homology blocks II and III defined in Fig. 2, lane 5). Percentage of input protein bound in lanes $1-5$ was $8 \%, 2 \%, 6 \%, 41 \%$, and $0 \%$, respectively. Although the very weak interaction between TBP and the BRF zinc finger region in lane 2 is likely to be artifactual, we cannot exclude the possibility that it is of physiological significance. $(C)$ BRF derivatives that do not bind TBP are expressed at high levels in soluble form and are thus unlikely to be folded aberrantly. Portions of GST-agarose beads bearing purified derivatives 1-89 (lane 1), 263-431 (lane 2), or 263-596 (lane 3) were electrophoresed on a 10\% SDS-polyacrylamide gel and detected by Coomassie staining. The positions of the derivatives are indicated by arrows.

terminal 165 amino acid residues of BRF, including homology blocks II and III, abolishes binding of the carboxy-terminal half of BRF to TBP (Fig. 4B, lane 5). Consistent with this result, previous studies have indicated that deletions of the carboxy-terminal region abrogate BRF function in vivo (Buratowski and Zhou 1992; Colbert and Hahn 1992). We speculate that the inability to currectly transcribe class III genes in yeast strains bearing these carboxy-terminal BRF deletions may be a consequence of a TBP-BRF interaction defect.

\section{Interactions between BRF and a subunit of Pol III}

BRF has also been hypothesized to act in the recruitment of the Pol III enzyme to class III genes. The $34-\mathrm{kD}$ subunit (C34), a subunit that is unique to Pol III, is known from photoaffinity DNA cross-linking studies to lie directly downstream from BRF in the assembled Pol III transcription complex (Bartholomew et al. 1993). To see whether BRF can interact specifically with C34, we again employed the GST-BRF pulldown assay. As shown in Figure 6A, C34 binds to fusions bearing both fulllength BRF and the amino-terminal half of this protein (lanes 1 and 2, respectively). In contrast, essentially no binding is observed to either GST alone (lane 6) or to a fusion containing the carboxy-terminal half of BRF (lane 5). When we divided the amino-terminal half of BRF into its zinc finger and direct repeat regions, we found that the latter, but not the former, can bind C34 effectively (Fig 6A, lanes 3 and 4, respectively). Furthermore, when each of the two BRF repeat elements was tested sepa- rately, C34 bound well to the first but not the second (data not shown). We therefore conclude that the direct repeat region of BRF interacts specifically with C34 and that this interaction appears to be mediated primarily by the first repeat. To address which regions of $\mathrm{C} 34$ are involved in specifying the BRF interaction, we generated a version of C34 in which the carboxy-terminal 120 amino acid residues had been deleted and tested it for BRF binding (Fig. 6B). It was found to be unable to interact with BRF, indicating that the carboxyl terminus of $\mathrm{C} 34$ is required for BRF binding.

It is well established that TFIIB binds Pol II (Ha et al. 1993). Because the amino-terminal half of BRF that binds the C34 subunit of Pol III is homologous in sequence to TFIIB, we wondered whether TFIIB might also be able to bind this protein. To test this, we synthesized $S$. cerevisiae TFIIB as a GST fusion and tested for its ability to interact with $\mathrm{C} 34$. This test revealed that $\mathrm{C} 34$ can interact efficiently with BRF but not TFIIB (Fig. 6C, lanes 1 and 2 , respectively|. These findings suggest that although TFIIB and BRF are related in sequence, only BRF can associate with the Pol III-specific subunit C34. This specificity of interaction may, at least in part, be responsible for the ability of class III transcription complexes to selectively recruit the appropriate RNA polymerase.

\section{Discussion}

In this paper we have shown by protein-protein interaction assays that BRF binds specifically to several components of the Pol III transcriptional apparatus. Consis- 


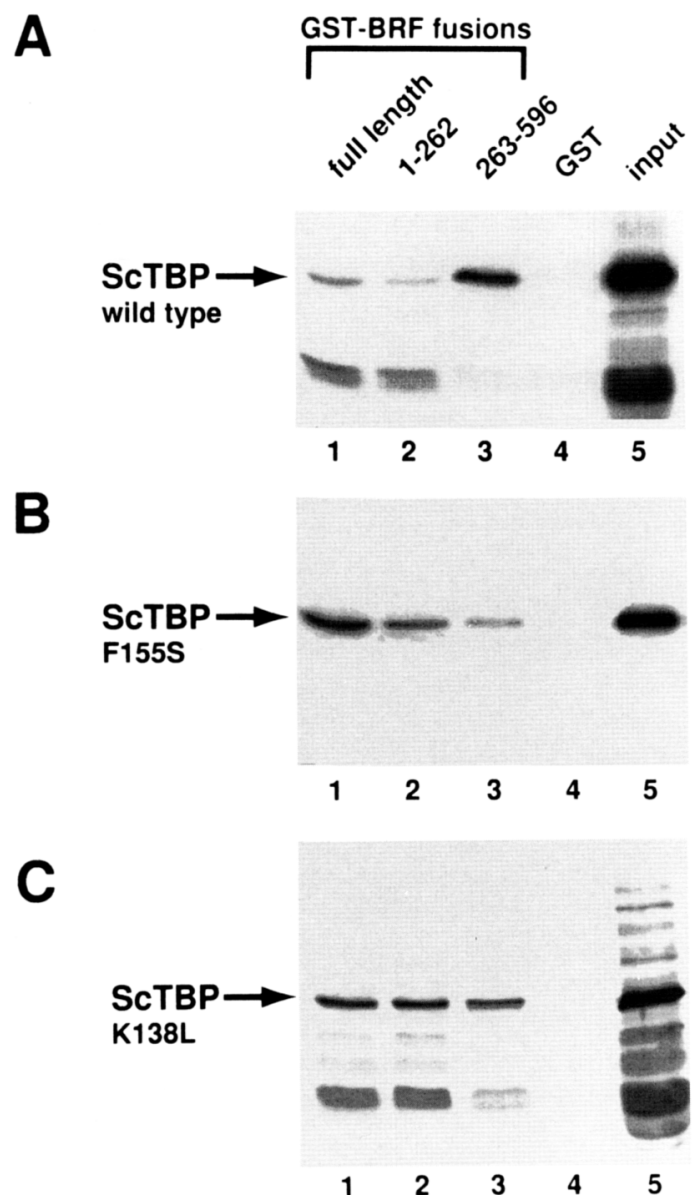

Figure 5. Mutations of TBP that impair Pol III transcription specifically abrogate interactions with the BRF carboxy-terminal region. ${ }^{35} \mathrm{~S}$-Labeled in vitro-translated derivatives of $S$. cerevisiae TBP were assayed for binding to various GST-BRF fusions as described in Fig. 4. The assays presented here were all performed simultaneously. (A) Wild-type TBP was tested for interactions with GST fusions to full-length BRF (lane 1), the amino-terminal half of BRF (residues 1-262; lane 2), the carboxy-terminal half of BRF (residues 263-596; lane 3), or GST alone (lane 4). Wild-type TBP binds to the carboxy-terminal portion of BRF strongly (lane 3) and also to the BRF amino-terminal region (lane 2). Percentage of input protein bound in lanes 1-4 was $5 \%, 1 \%, 35 \%$, and $0 \%$, respectively. $(B)$ The Pol III-specific TBP mutation F155S reduces binding to the carboxy-terminal half of BRF (lane 3), whereas binding to full-length BRF (lane 1) and the BRF amino-terminal region (lane 2) is actually increased. Percentage of input protein bound in lanes 1-4 was $26 \%, 6 \%, 5 \%$, and $0 \%$, respectively. $(C)$ The Pol III-specific TBP mutation $\mathrm{K} 138 \mathrm{~L}$ reduces binding to the carboxy-terminal half of BRF but does not affect interactions with the amino-terminal region of BRF significantly. Percentage of input protein bound in lanes $1-4$ was $34 \%, 30 \%, 17 \%$, and $1 \%$, respectively.

tent with TBP and BRF being subunits of TFIIIB, we find that these proteins interact with one another. We have also shown that BRF binds to the $\tau 135$ component of TFIIIC and to the C34 subunit of Pol III. Previous protein-DNA cross-linking studies have indicated that BRF is located near both $\tau 135$ and C34 in assembled Pol III transcription complexes (Bartholomew et al. 1991, 1993). It is therefore likely that the interactions we observe in vitro are relevant functionally. Together with previous

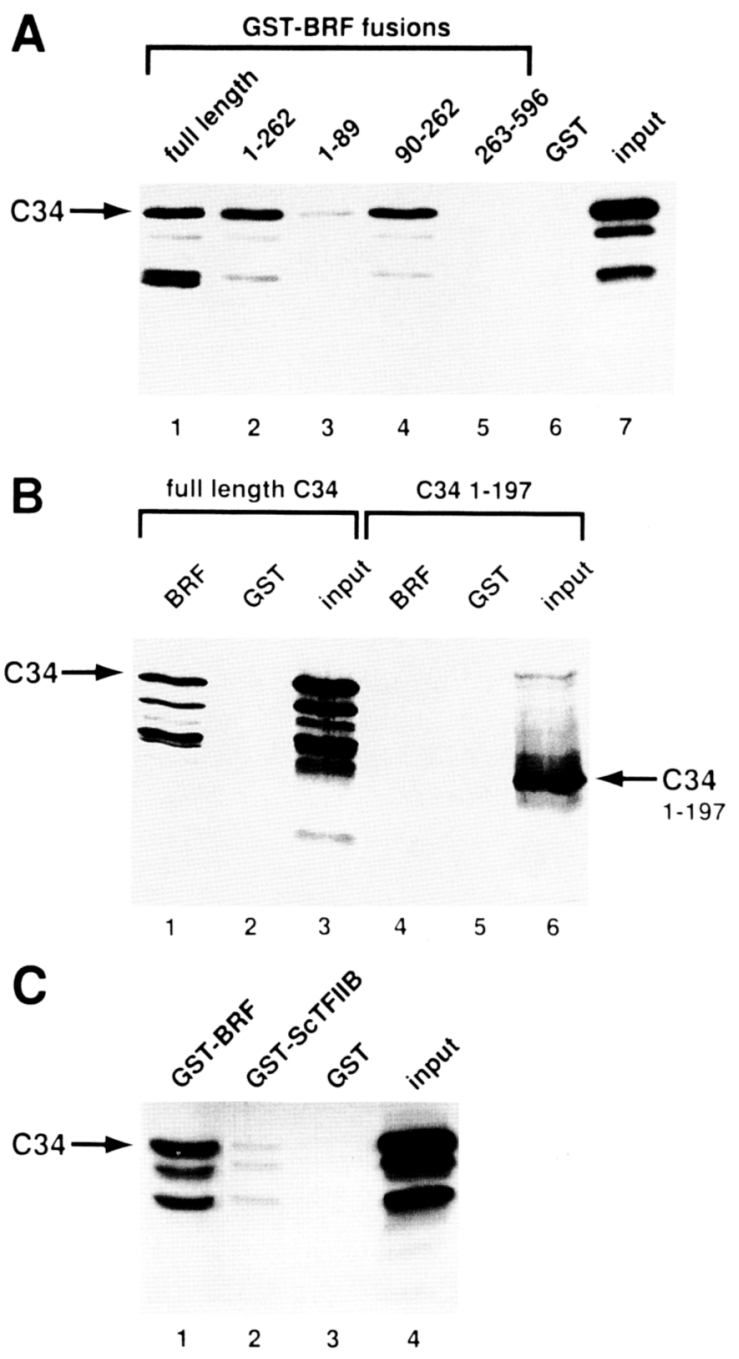

Figure 6. (A) BRF binds specifically to the C34 subunit of Pol III. ${ }^{35} \mathrm{~S}$-Labeled in vitro-translated C34 subunit of S. cerevisiae Pol III was tested for binding to GST fusions of the following: full-length BRF (lane 1), BRF residues 1-262 (zinc finger together with direct repeat region; lane 2), BRF residues 1-89 (zinc finger region alone; lane 3), BRF residues 90-262 (direct repeat region alone; lane 4), BRF residues 263-596 (carboxy-terminal nonTFIIB-homologous domain; lane 5), or GST alone (lane 6). Amount of input C34 is shown to allow assessment of binding efficiencies. Percentage of input protein bound in lanes 1-6 was $34 \%, 33 \%, 2 \%, 34 \%, 0 \%$, and $0 \%$, respectively. $(B)^{35} \mathrm{~S}$-Labeled in vitro-translated wild-type C34 (lanes 1-3) or C34 residues 1-197 (lanes 4-6) were tested for binding to GST fused to fulllength BRF (lanes 1,4), or to GST alone (lanes 2,5). Percentage of input protein bound was $41 \%, 0 \%, 0 \%$, and $0 \%$ for lanes $1,2,4$, 5 , respectively. $(C){ }^{35} \mathrm{~S}$-Labeled C34 was tested for binding to GST fusions of the following: full-length BRF (lane 1), S. cerevisiae TFIIB (lane 2), or GST alone (lane 3). Percentage of input protein bound in lanes $1-3$ was $35 \%, 0 \%$, and $2 \%$, respectively. 
observations, our data support a model in which BRF plays a central role in Pol III transcription. First, BRF forms part of TFIIIB by interacting with TBP and possibly the B" component of this Pol III-specific TBP-TAF complex (Kassavetis et al. 1991, 1992). Second, by binding to the $\tau 135$ subunit of DNA-bound TFIIIC, BRF mediates the recruitment of TFIIIB to class III templates. Third, BRF confers Pol III specificity to the transcription complex by interacting specifically with the C34 subunit of Pol III.

Through cloning BRF homologs from $K$. lactis and $C$. albicans and comparing their sequences with $S$. cerevisiae BRF, we have defined evolutionarily conserved regions of BRF that may correspond to important functional domains. Although $K$. lactis and C. albicans are both yeasts, they are distant from $S$. cerevisiae from an evolutionary standpoint. For example, K. lactis is estimated by rRNA sequence comparisons to have diverged from $S$. cerevisiae $\sim 10^{8}$ years ago and the evolutionary distance between S. cerevisiae and C. albicans is considerably larger still (Barns et al. 1991; Van de Peer et al. 1992). Strikingly, the BRF proteins of $K$. lactis and $C$. albicans are $62 \%$ and $47 \%$ identical, respectively, to $S$. cerevisiae BRF. This conservation is much higher than that of gene-specific regulatory factors such as GAL4/ LAC9 and heat shock factor (HSF), where homologies between the $K$. lactis and S. cerevisiae proteins are confined to relatively short regions (Salmeron and Johnston 1986; Wray et al. 1987; Jakobsen and Pelham 1991). The level of evolutionary conservation of BRF is similar to that of TFIIB, which is $67 \%$ identical between $K$. lactis and S. cerevisiae (Na and Hampsey 1993). (The aminoterminal portion of BRF that is related in sequence to TFIIB is actually more conserved from $S$. cerevisiae to $K$. lactis than are the TFIIB proteins of these two species.) The strong evolutionary conservation of BRF is consistent with this protein's role as the linchpin of the Pol III transcriptional apparatus.

From the three-way comparison of BRF proteins in Figure $2 \mathrm{~A}$, we can divide $\mathrm{BRF}$ tentatively into four highly conserved domains (Fig. 2B). The first lies at the extreme amino terminus and includes a cysteine-rich sequence postulated to form a zinc-binding "finger" structure (Buratowski and Zhou 1993; Ha et al. 1993). This region is well conserved in $K$. lactis and C. albicans BRFs, suggesting an important role. Deleting this region of S. cerevisiae BRF is lethal (Colbert and Hahn 1992). Because the analogous TFIIB domain is implicated in binding TFIIF (Barberis et al. 1993; Buratowski and Zhou 1993; Ha et al. 1993), this region of BRF might interact with a Pol III basal factor with homology to TFIIF.

The second conserved region of BRF encompasses the two imperfect direct repeats of $\sim 76$ amino acid residues that are related to the repeat elements of TFIIB. In this region, $73 \%$ of the residues are identical in all three BRF proteins in Figure 2A, indicating that it is particularly crucial for BRF function. Our data suggest that the conservation of this domain reflects the fact that it mediates important interactions with several components of the Pol III transcription apparatus. One function of this re- gion appears to be to interact with TBP, as we have shown that a GST fusion containing this domain binds TBP specifically in vitro. Consistent with this result, recent work has demonstrated that the analogous repeat region of TFIIB also binds TBP (Barberis et al. 1993; Ha et al. 1993; Hisatake et al. 1993; Yamashita et al. 1993).

We have also demonstrated that the BRF direct repeat region and the first BRF repeat in isolation can interact with the 34-kD subunit of Pol III. This is in line with previous studies that have suggested that BRF is involved in Pol III recruitment (Bartholomew et al. 1993) and with the fact that the homologous repeat region of TFIIB binds Pol II (Ha et al. 1993). It has been speculated previously that C34 might interact with class III transcription factors on the basis of the fact that antibodies against C34 inhibit specific transcription but not factorindependent nonspecific transcription (Huet et al. 1985). Furthermore, it has been shown that BRF interacts with C34 using a yeast two-hybrid assay (Werner et al. 1993), although this approach could not exclude the possibility of an indirect interaction. It should be noted, however, that interactions between BRF and Pol III may not be sufficient for efficient Pol III recruitment, because the 90-kD component of TFIIIB $\left(\mathrm{B}^{\prime \prime}\right)$ is necessary for transcription to proceed (Kassavetis et al. 1991, 1992). By synthesizing a deletion derivative of C34, we have shown that its carboxy-terminal 120 amino acid residues are required for effective BRF binding. This tallies with the observation that the carboxy-terminal region of C34 is essential for yeast viability (Stettler et al. 1992). Importantly, unlike several Pol III subunits, C34 is not present in either of the other two nuclear pols. The interaction between BRF and C34 may therefore play a crucial role in defining the pol specificity of Pol III transcription preinitiation complexes. In line with this prediction, we find that the Pol II general factor TFIIB is unable to bind C34 effectively. Perhaps TFIIB interacts with a Pol II-specific subunit that performs an analogous function to C34.

Other possible roles for the direct repeat domain of BRF include binding to DNA and interacting with Pol III regulatory factors. Previous work has identified a region at the end of the first TFIIB repeat that contains a cluster of basic residues and is predicted to form an amphipathic $\alpha$-helix. This region has been implicated in mediating TFIIB interactions with TBP and upstream regulators such as GAL4-VP16, and point mutagenesis has suggested that the basic residues are crucial for TFIIB function (Lin et al. 1991; Ha et al. 1993; Roberts et al. 1993; Yamashita et al. 1993). Interestingly, the analogous region of BRF constitutes the most extensive region of homology between the three BRF proteins in Figure 2A (indicated by a broken underline), although the conservation with TFIIB here is marginal. It is tempting to speculate that this part of BRF might constitute an interaction surface for TBP, C34, and/or Pol III regulatory factors.

The remaining conserved regions of BRF lie within the carboxy-terminal portion of the protein that has no homology to TFIIB. The most obvious feature of this do- 
main is its high charge density; for example, in $K$. lactis $\mathrm{BRF}, 20 \%$ of the residues in this region are acidic and $19 \%$ are basic. Overall, this part of BRF is less conserved than the amino-terminal region /the carboxyl termini of $K$. lactis and C. albicans proteins are $51 \%$ and $31 \%$ identical, respectively, to $S$. cerevisiae BRF|, and several gaps must be introduced to optimize the alignments. Nevertheless, three units of strong homology can be discerned readily (indicated I, II, and III in Fig. 2, A and B). The longest of these is region II, which covers 77 amino acid residues and is $80 \%$ identical between $S$. cerevisiae and K. lactis and $43 \%$ identical between $S$. cerevisiae and C. albicans.

The surprising demonstration that the carboxy-terminal half of BRF associates strongly with TBP suggests that this interaction may be of importance to Pol III transcription. Significantly, we find that deletion of homology blocks II and III of the BRF carboxy-terminal region abrogates the binding of this domain to TBP. Interestingly, previous studies have indicated that deletion of the carboxy-terminal 35-100 residues of BRF that span homology blocks II and III disrupts BRF function in vivo (Buratowski and Zhou 1992; Colbert and Hahn 1992). It is tempting to speculate, therefore, that the Pol III transcription defect in yeast strains bearing these mutations is a result of the inability of the carboxy-terminal half of BRF to bind TBP.

Using a regional codon randomization strategy, Cormack and Struhl (1993) recently generated a large number of single amino acid residue mutations in TBP and tested them for their effects on TBP function in vivo. The majority either had no discernible phenotype or had generalized effects on transcription. However, of the 186 mutations examined, 65 were specifically defective in Pol III transcription and $85 \%$ of these mapped to an exposed surface of TBP forming a groove across the upper part of the protein. Kim and Roeder (1994) have also identified a Pol III-specific TBP mutation (K138L) that maps to this vicinity. In all cases tested, overexpression of BRF in strains bearing these Pol III-specific TBP mutations results in partial reversal of the growth defect (Cormack and Struhl 1993). This suggests that BRF interacts with the region of TBP defined by the Pol IIIspecific mutations.

Because the amino-terminal half of BRF is highly related to TFIIB, one would anticipate that these two regions would make similar contacts with TBP. If the TFIIB homologous region were the only region of BRF to interact with TBP, one would therefore expect that TBP mutations specifically affecting binding to TFIIB would arise at a similar frequency to those affecting interactions with BRF. This is not the case, however; the mutagenesis procedure of Cormack and Struhl (1993) obtained 65 TBP mutants that were Pol III specific but only 10 that were Pol II specific. Our data showing that the carboxyl terminus of BRF also contacts TBP and that Pol III-specific TBP mutants are defective in this interaction help resolve this enigma. Specifically, our data suggest that in addition to the amino-terminal half of BRF binding TBP in an analogous fashion to TFIIB, the carboxyl terminus of BRF mediates interactions with the Pol IIIspecific surface of TBP.

BRF therefore appears to possess two distinct TBP-interacting domains. Although it is not yet clear why BRF has evolved in this way, it can be surmised that the two regions will perform different functions in Pol III transcription complex assembly. Interestingly, full-length BRF binds to TBP less effectively than the carboxy-terminal region alone and does so with a comparable efficacy to the amino-terminal BRF domain alone. This suggests that in the complete BRF molecule, the carboxyterminal TBP-binding region is not normally accessible. It is tempting to speculate that initial binding of BRF to TBP is mediated by the TFIIB homologous domain of BRF. Subsequently, the carboxy-terminal region of BRF may become exposed by a conformational shift, perhaps induced by the binding of factors such as the $135-\mathrm{kD}$ component of TFIIIC, the other TFIIIB subunit $\mathrm{B}^{\prime \prime}$, Pol III, or regulatory proteins. Previous studies have revealed conformational shifts in BRF during Pol III transcription complex assembly (Kassavetis et al. 1992). This type of mechanism may ensure an ordered assembly of TFIIIB on the promoter and might preclude the formation of nonproductive interactions between BRF and other Pol III transcription factors in inappropriate non-promoterbound contexts.

\section{Materials and methods \\ Cloning, analysis, and expression of BRF proteins}

Standard molecular cloning techniques were as described by Ausubel et al. (1991). Dideoxy chain-termination sequencing was performed using the Sequenase version 2.0 system (U.S. Biochemical) according to the manufacturer's recommendations. Sequence analysis was by the MacVector program package (International Biotechnologies, Inc., New Haven, CT) and by the program CLUSTAL V for multiple alignments (Higgins et al. 1992). Low-stringency PCR reactions were performed as described previously (Rowlands et al. 1994). PCR products were cloned into pBluescript $\mathrm{KS} \mid+1$.

\section{Cloning and expression of GST-BRF and GST-TFIIB fusions}

Full-length $S$. cerevisiae BRF was cloned by amplifying the gene from genomic DNA using the following oligonucleotides (all oligonucleotides read $5^{\prime} \rightarrow 3^{\prime}$ ): GTCCATCGATATGCCAGTGTGTAAGAACTGT and GTCCATCGATCCTAAACAAACCGTCAATGGC. The $1.8-\mathrm{kb}$ fragment thus generated was cut with ClaI and ligated into the ClaI site of pBluescript $\mathrm{KS}(+)$ (Stratagene) as well as into the ClaI site of the vector pGEX-20 (R. Treisman, unpubl.) prepared from the E. coli $\mathrm{dam}^{-} \mathrm{dcm}^{-}$ strain GM272. To generate the 1-262 and 263-596 constructs, a full-length $1.8-\mathrm{kb}$ fragment was also generated from genomic DNA using oligonucleotides as above, except that the $5^{\prime}$ oligonucleotide contained an EcoRI site (vide infra). This fragment was cut with EcoRI and ClaI to generate a 0.8 -kb fragment (cut at the natural EcoRI site within the BRF gene) encoding residues $1-262$ and a $1.0-\mathrm{kb}$ fragment encoding the rest of BRF. The 0.8 -kb fragment was ligated into EcoRI-cut pGEX-2TK (Pharmacia); the $1.0-\mathrm{kb}$ fragment was ligated into $E c o \mathrm{RI}-C l a I$-cut pGEX20. The other constructs were generated by PCR cloning from the pBS KS $|+|$ BRF construct into the EcoRI site of pGEX-2TK, 
using the appropriate oligonucleotides: BRF 1-89, TGACGAATTCGAAT GCCAGTGTGTAAGAACTGT and TGACGAATTCGGCGCGTAATTTCCTTCTTGC; and BRF 90-262, TGACGAATTCGCGTTTCTTACGCATTACAT and TGACGAATTCGTTCAACCGTTGCTG. BRF 263-431 was constructed by cutting pGEX-20 BRF 263-596 with XbaI and religating the $5.5-\mathrm{kb}$ fragment thus generated. The GST-ScTFIIB construct was made in a similar fashion by PCR from genomic DNA with the following oligonucleotides: TAGAGAATTCGAATGATGACTAGGGAGAGCATA and TATCGAATTCTTATTTCTTTTCAACGCCCGGTA and cloned into the EcoRI site of pGEX-2TK.

\section{Cloning and expression of other factors}

Each $S$. cerevisiae transcription factor $(\tau 135$, RNA Pol III C34 subunit, or TBP) was cloned using the following oligonucleotides to amplify the gene by PCR from genomic DNA; TFIIIC 135-kD 1-363, TGTAAGATCTATGGCAGCAGGAAAATTGAAAAAG and AGAACTGCAGTCAGAGAATGTTCAAAGAAGACCAGTC; TFIIIC 135-kD 364-714, TTTGAGATCTATGGCGGAACTTTTCTTAAAACTCGCA and CTCTCTGCAGTCATAGCGGCCCTTCAAACACACTATC; TFIIIC 135-kD 715-1025, AGGGAGATCTATGGAGGAAAGAGTAACCTTAACT and AAACCTGCAGTCAAATACTTAAATACTTCTCCAT; Pol III subunit C34, TCGACAGCTGATGAGTGGAATGATAGAAAATGGG and AAAACTGCAGTCAAATTGTCCATTCATCGAAATA; and TBP, TTTTCAGCTGATGGCCGATGAGGAACGTTTAAAG and TCCTCTGCAGTCACATTTTTCTAAATTCACTTAG.

The resulting fragments were then ligated into appropriately digested ping 14 in vitro transcription-translation vector (Bannister et al. 1991), and the resulting construct was translated and radiolabeled with $\left[{ }^{35} S \mid\right.$ methionine using the TNT rabbit reticulocyte lysate coupled transcription-translation system (Promega) according to the manufacturer's instructions. Radiolabeled human TBP was expressed from the construct pARhTFIID (GPP-63), a generous gift of M.G. Peterson (Peterson et al. 1990). The missense mutants of ScTBP were constructed using oligonucleotide-directed site mutagenesis of a uracil-containing single-stranded DNA (ssDNA) template produced from the ping 14-ScTBP construct, essentially as in Ausubel et al. (1991). The carboxy-terminal deletion of C34 (1197) was synthesized by linearizing the ping 14-C34 template with BstBI. This linearized template was then transcribed and translated to generate labeled protein in a conventional twostep in vitro translation reaction (Ausubel et al. 1991).

\section{Protein-protein interaction studies}

Generation and purification of fusion proteins consisting of BRF domains linked to GST was accomplished by the method of Smith and Johnson (Smith and Johnson 1988; Lin and Green 1991; Bannister et al. 1993\}. Fusion proteins were purified by adsorption onto glutathione-agarose beads (Smith and Johnson 1988; Bannister et al. 1993) and analyzed by SDS-PAGE and Coomassie staining. Equal amounts of each protein were used in the protein-protein interaction studies, except for unfused GST, which was used, at least, at a concentration fivefold higher. Identical negative results were also obtained when lower amounts of unfused GST were employed. Interaction assays were conducted as follows: $10-\mu l$ samples of glutathioneSepharose beads bearing either GST alone or the GST-BRF fusion were preincubated at room temperature for $15 \mathrm{~min}$ on a rotating wheel with $30 \mu \mathrm{g}$ of BSA in buffer $Z^{\prime} 200 / 25 \mathrm{~mm}$ HEPES-KOH at $\mathrm{pH} 7.5,200 \mathrm{mM} \mathrm{KCl}, 12.5 \mathrm{~mm} \mathrm{MgCl}_{2}, 20 \%$ glycerol, $0.1 \% \mathrm{NP}-40,20 \mu \mathrm{M} \mathrm{ZnSO}_{4}, 2 \mathrm{~mm}$ DTT). A sample of radiolabeled in vitro-translated protein was then added, and incubation continued for another $60 \mathrm{~min}$. After extensive washing with buffer NETN/200 (20 mM Tris- $\mathrm{HCl}$ at $\mathrm{pH} 8.0,200 \mathrm{~mm}$ $\mathrm{NaCl}, 1 \mathrm{~mm}$ EDTA, $0.5 \% \mathrm{NP}-40$ ), the agarose beads were mixed with SDS sample buffer and boiled; then samples were analyzed by SDS-PAGE followed by fluorography. All assays were repeated multiple times with at least two different preparations of fusion protein. Quantitations were carried out from autoradiographs using a Quantimet (Cambridge Instruments).

\section{Yeast transformation and complementation}

A S. cerevisiae strain (rad3-52; leu2-3,112; his3 $\Delta 200$; $t d s 4 \Delta:: L E U 2$ ) containing a plasmid bearing the HIS3 gene and BRF temperature-sensitive allele $t d s 4-144$ (restrictive temperature $38^{\circ} \mathrm{C}$ ) was provided by S. Buratowski (Whitehead Institute, Cambridge, MA). Transformation with plasmids containing the $U R A 3$ gene and either CaBRF or the wild-type $S$. cerevisiae $\mathrm{BRF} / \mathrm{TDS} 4$ was performed using the lithium acetate procedure of Schiestl and Gietz (1989). To measure growth rates of resulting strains, cells were grown at the permissive temperature to saturation and then diluted to the same $\mathrm{OD}_{650}$ values in $20 \mathrm{ml}$ of $\mathrm{YNB} / 2 \%$ glucose supplemented with $20 \mu \mathrm{g} / \mathrm{ml}$ of uracil prewarmed to the appropriate temperature. Aliquots of the cultures were taken for measurement of culture density by $\mathrm{OD}_{650}$ at intervals.

\section{Acknowledgments}

We thank S. Buratowski for providing the tsBRF $S$. cerevisiae strain and plasmid containing $S$. cerevisiae BRF, R. Hather for providing the $K$. lactis genomic library, S. Sen Gupta and D. Kerridge for the C. albicans library, R. Treisman for pGEX-20, K. Struhl for Pol III-specific TBP mutants, and I. Willis for communicating unpublished data. Thanks also go to $\mathrm{R}$. White for his critical comments and to Ruth Dendy for assistance in preparing the manuscript. B.K. is a member of the University of Cambridge School of Medicine's M.B./Ph.D. program and is supported by a Wellcome Trust Prize studentship. The S.P.J. laboratory is funded principally by project grant SP2143/0101 from the Cancer Research Campaign.

The publication costs of this article were defrayed in part by payment of page charges. This article must therefore be hereby marked "advertisement" in accordance with 18 USC section 1734 solely to indicate this fact.

\section{References}

Ausubel, F.M., R. Brent, R.E. Kingston, D.D. Moore, J.G. Seidman, J.A. Smith, and K. Struhl. 1991. In Current protocols in molecular biology. John Wiley and Sons/Greene, New York.

Bannister, A.J., A. Cook, and T. Kouzarides. 1991. In vitro DNA binding activity of Fos/Jun and BZLF1 but not C/EBP is affected by redox changes. Oncogene 6: 1243-1250.

Bannister, A.J., T.M. Gottlieb, T. Kouzarides, and S.P. Jackson. 1993. c-Jun is phosphorylated by the DNA-dependent protein kinase in vitro; definition of the minimal kinase recognition motif. Nucleic Acids Res. 21: 1289-1295.

Barberis, A., C.W. Muller, S.C. Harrison, and M. Ptashne. 1993. Delineation of two functional regions of transcription factor TFIIB. Proc. Natl. Acad. Sci. 90: 5628-5632.

Barns, S.M., D.J. Lane, M.L. Sogin, C. Bibeau, and W.G. Weisberg. 1991. Evolutionary relationships among pathogenic Candida species and relatives. J. Bacteriol. 173: 2250-2255. 
Bartholomew, B., G.A. Kassavetis, and E.P. Geiduschek. 1991. Two components of Saccharomyces cerevisiae transcription factor IIIB (TFIIIB) are stereospecifically located upstream of a tRNA gene and interact with the second-largest subunit of TFIIIC. Mol. Cell. Biol. 11: 5181-5189.

Bartholomew, B., D. Durkovich, G.A. Kassavetis, and E.P. Geiduschek. 1993. Orientation and topography of RNA polymerase III in transcription complexes. Mol. Cell. Biol. 13: 942-952.

Buratowski, S. and H. Zhou. 1992. A suppressor of TBP mutations encodes an RNA polymerase III transcription factor with homology to TFIIB. Cell 71: 221-230.

. 1993. Functional domains of transcription factor TFIIB. Proc. Natl. Acad. Sci. 90: 5633-5637.

Chiang, C.-M., H. Ge, Z. Wang, A. Hoffmann, and R.G. Roeder. 1993. Unique TATA-binding protein-containing complexes and cofactors involved in transcription by RNA polymerases II and III. EMBO I. 12: 2749-2762.

Colbert, T. and S. Hahn. 1992. A yeast TFIIB-related factor involved in RNA polymerase III transcription. Genes \& Dev. 6: 1940-1949.

Comai, L., N. Tanese, and R. Tjian. 1992. The TATA-binding protein and associated factors are integral components of the RNA polymerase I transcription factor, SL1. Cell 68: 965976.

Cormack, B.P. and K. Struhl. 1993. Regional codon randomization: Defining a TATA-binding protein surface required for RNA polymerase III transcription. Science 262: 244-248.

Dynlacht, B.D., T. Hoey, and R. Tjian. 1991. Isolation of coactivators associated with the TATA-binding protein that mediate transcriptional activation. Cell 66: 563-576.

Eberhard, D., L. Tora, J.-M. Egly, and I. Grummt. 1993. A TBPcontaining multiprotein complex (TIF-IB) mediates transcription specificity of murine RNA polymerase I. Nucleic Acids Res. 21: 4180-4186.

Geiduschek, E.P. and G.P. Tocchini-Valentini. 1988. Transcription by RNA polymerase III. Annu. Rev. Biochem. 57: 873914.

Goebl, M. and M. Yanagida. 1991. The TPR snap helix: A novel protein repeat motif from mitosis to transcription. Trends Biochem. Sci. 16: 173-177.

Ha, I., S. Roberts, E. Maldonado, X. Sun, L.-U. Kim, M. Green, and D. Reinberg. 1993. Multiple functional domains of human transcription factor IIB: Distinct interactions with two general transcription factors and RNA polymerase II. Genes \& Dev. 7: 1021-1032.

Hernandez, N. 1993. TBP, a universal eukaryotic transcription factor? Genes \& Dev. 7: 1291-1308.

Higgins, D.G., A.J. Bleasby, and R. Fuchs. 1992. CLUSTAL V: Improved software for multiple sequence alignment. Comp. Appl. Biosci. 8: 189-191.

Hisatake, K., R.G. Roeder, and M. Horikoshi. 1993. Functional dissection of TFIIB domains required for TFIIB-TFIID-promoter complex formation and basal transcription activity. Nature 363: 744-747.

Huet, J. and A. Sentenac. 1993. The TATA-binding protein participates in TFIIIB assembly on tRNA genes. Nucleic Acids Res. 20: 6451-6454.

Huet, J., M. Riva, A. Sentenac, and P. Fromageot. 1985. Yeast RNA polymerase $C$ and its subunits. Specific antibodies as structural and functional probes. I. Biol. Chem. 260: 1530415310.

Jakobsen, B.K. and H.R.B. Pelham. 1991. A conserved heptapeptide restrains the activity of the yeast heat shock transcription factor. $E M B O J .10: 369-375$.

Kao, C.C., P.M. Lieberman, M.C. Schmidt, Q. Zhou, R. Pei, and
A.J. Berk. 1990. Cloning of a transcriptionally active human TATA binding factor. Science 248: 1646-1650.

Kassavetis, G.A., D.L. Riggs, R. Negri, L.H. Nguyen, and E.P. Geiduschek. 1989. Transcription factor IIIB generates extended DNA interactions in RNA polymerase III transcription complexes on tRNA genes. Mol. Cell. Biol. 9: 25512566.

Kassavetis, G.A., B.R. Braun, L.H. Nguyen, and E.P. Geiduschek. 1990. S. cerevisiae TFIIIB is the transcription initiation factor proper of RNA polymerase III, while TFIIIA and TFIIIC are assembly factors. Cell 60: 235-245.

Kassavetis, G.A., B. Bartholomew, J.A. Blanco, T.E. Johnson, and E.P. Geiduschek. 1991. Two essential components of the Saccharomyces cerevisiae transcription factor TFIIIB: transcription and DNA-binding properties. Proc. Natl. Acad. Sci. 88: 7308-7312.

Kassavetis, G.A., C.A.P. Joazeiro, M. Pisano, E.P. Geiduschek, T. Colbert, S. Hahn, and J.A. Blanco. 1992. The role of the TATA-binding protein in the assembly and function of the multisubunit yeast RNA polymerase III transcription factor, TFIIIB. Cell 71: 1055-1064.

Kim, T.K. and R.G. Roeder. 1994. Involvement of the basic repeat domain of TATA-binding protein (TBP) in transcription by RNA polymerases I, II, and III. J. Biol. Chem. 269: 48914894.

Kim, Y., J.H. Geiger, S. Hahn, and P.B. Sigler. 1993. Crystal structure of a yeast TBP/TATA-box complex. Nature 365: 512-520.

Kyte, J. and R.F. Doolittle. 1982. A simple method for displaying the hydropathic character of a protein. I. Mol. Biol. 157: 105132.

Lin, Y.-S. and M.R. Green. 1991. Mechanism of activation of an acidic transcriptional activator in vitro. Cell 64: 971-981.

Lin, Y.-S., I. Ha, E. Maldonado, D. Reinberg, and M.R. Green. 1991. Binding of general transcription factor TFIIB to an acidic activating region. Nature 353: 569-571.

Lobo, S.M., M. Tanaka, M.L. Sullivan, and N. Hernandez. 1992. A TBP complex essential for transcription from TATA-less but not TATA-containing RNA polymerase III promoters is part of the TFIIIB fraction. Cell 71: 1029-1040.

Lopez-de-Leon, A., M. Librizzi, K. Tuglia, and I. Willis. 1992. PCF4 encodes an RNA polymerase III transcription factor with homology to TFIIB. Cell 71: 211-220.

Malik, S., D.K. Lee, and R.G. Roeder. 1993. Potential RNA polymerase II-induced interactions of transcription factor TFIIB. Mol. Cell. Biol. 13: 6253-6259.

Marck, C., O. Lefebvre, C. Carles, M. Riva, N. Chaussivert, A. Ruet, and A. Sentenac. 1993. The TFIIIB-assembling subunit of yeast transcription factor TFIIIC has both tetratricopeptide repeats and basic helix-loop-helix motifs. Proc. Natl. Acad. Sci. 90: 4027-4031.

Na, J.G. and M. Hampsey. 1993. The Kluyveromyces gene encoding the general transcription factor IIB: Structural analysis and expression in Saccharomyces cerevisiae. Nucleic Acids Res. 21: 3413-3417.

Peterson, M.G., N. Tanese, B.F. Pugh, and R. Tjian. 1990. Functional domains and upstream activation properties of cloned human TATA binding protein. Science 248: 1625-1630.

Pinto, I., D.E. Ware, and M. Hampsey. 1992. The yeast SUA7 gene encodes a homolog of human transcription factor TFIIB and is required for normal start site selection in vivo. Cell 68: $977-988$.

Pugh, B.F. and R. Tjian. 1992. Diverse transcriptional functions of the multisubunit eukaryotic TFIID complex. I. Biol. Chem. 267: 679-682.

Rigby, P.W.J. 1993. Three in one and one in three: It all depends on TBP. Cell 72: 7-10. 
Roberts, S.G.E., I. Ha, E. Maldonado, D. Reinberg, and M.R. Green. 1993. Interaction between an acidic activator and transcription factor TFIIB is required for transcriptional activation. Nature 363: 741-744.

Rowlands, T., P. Baumann, and S.P. Jackson. 1994. The TATAbinding protein: A general transcription factor present in both eukaryotes and archaebacteria. Science 264: 13261329.

Salmeron, J.M. and S.A. Johnston. 1986. Analysis of the Kluyveromyces lactis positive regulatory gene LAC9 reveals functional homology to, but sequence divergence from, the Saccharomyces cerevisiae GAL4 gene. Nucleic Acids Res. 14: 7767-7781.

Schiestl, R.H. and R.D. Gietz. 1989. High efficiency transformation of intact yeast cells using single-stranded nucleic acids as a carrier. Curr. Genet. 16: 339-346.

Simmen, K.A., J. Bernues, J.D. Lewis, and I.W. Mattaj. 1992. Cofractionation of the TATA-binding protein with the RNA polymerase III transcription factor TFIIIB. Cell 20: 58895898.

Smith, D.B. and K.S. Johnson. 1988. Single-step purification of polypeptides expressed in Escherichia coli as fusions with glutathione S-transferase. Gene 67: 31-40.

Stettler, S., S. Mariotte, M. Riva, A. Sentenac, and P. Thuriaux. 1992. An essential and specific subunit of RNA polymerase III (C) is encoded by gene RPC34 in Saccharomyces cerevisiae. J. Biol. Chem. 267: 21390-21395.

Struhl, K. 1994. Duality of TBP, the universal transcription factor. Science 263: 1103-1104.

Taggart, A.K.P., T.S. Fisher, and B.F. Pugh. 1992. The TATAbinding protein and associated factors are components of Pol III transcription factor TFIIIB. Cell 71: 1015-1028.

Tschochner, H., M.H. Sayre, P.M. Flanagan, W.J. Feaver, and R.D. Kornberg. 1992. Yeast RNA polymerase II initiation factor e: Isolation and identification as the functional counterpart of human transcription factor IIB. Proc. Natl. Acad. Sci. 89: 11292-11296.

Van de Peer, Y., L. Hendricks, A. Goris, J. Neefs, M. Vancanneyt, K. Kersters, J. Berny, G.L. Hennebert, and R. de Wachter. 1992. Syst. Appl. Microbiol. 15: 250-258.

Wampler, S.L. and J.T. Kadonaga. 1992. Functional analysis of Drosophila transcription factor IIB. Genes \& Dev. 6: 15421552.

Werner, M., N. Chaussivert, I.M. Willis, and A. Sentenac. 1993. Interaction between a complex of RNA polymerase III subunits and the $70 \mathrm{kDa}$ component of transcription factor IIIB. I. Biol. Chem. 268: 20721-20724.

White, R.J. 1994. RNA polymerase III transcription in molecular biology intelligence unit. R.G. Landes Co., Austin, TX.

White, R.J. and S.P. Jackson. 1992a. Mechanism of TATA-binding protein recruitment to a TATA-less class III promoter. Cell 71: 1041-1053.

- 1992b. The TATA-binding protein: A central role in transcription by RNA polymerases I, II and III. Trends Genet. 8: $284-288$.

Willis, I.M. 1993. RNA polymerase III. Eur. J. Biochem. 212: 1-11.

Wray, L.V., M.M. Witte, R.C. Dickson, and M.I. Riley. 1987. Characterization of a positive regulatory gene, LAC9, that controls induction of the lactose-galactose regulon of Kluyveromyces lactis: Structural and functional relationships to GAL4 of Saccharomyces cerevisiae. Mol. Cell. Biol. 7: 1111-1121.

Yamashita, S., K. Hisatake, T. Kokubo, K. Doi, R.G. Roeder, M. Horikoshi, and Y. Nakatani. 1993. Transcription factor TFIIB sites important for interaction with promoter-bound TFIID. Science 261: 463-466. 


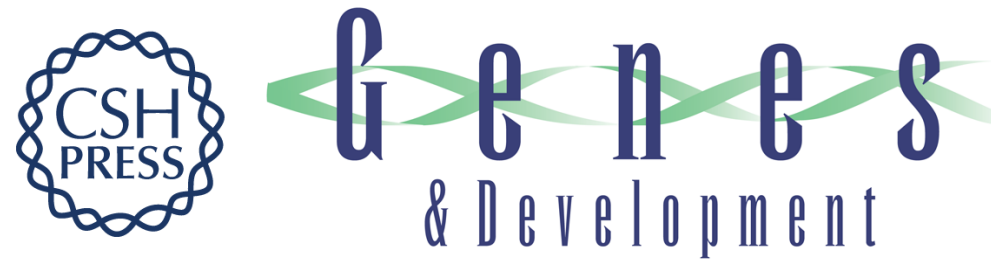

\section{Conserved functional domains of the RNA polymerase III general transcription factor BRF.}

B Khoo, B Brophy and S P Jackson

Genes Dev. 1994, 8:

Access the most recent version at doi:10.1101/gad.8.23.2879

References This article cites 60 articles, 26 of which can be accessed free at:

http://genesdev.cshlp.org/content/8/23/2879.full.html\#ref-list-1

License

Email Alerting

Service

Receive free email alerts when new articles cite this article - sign up in the box at the top right corner of the article or click here.

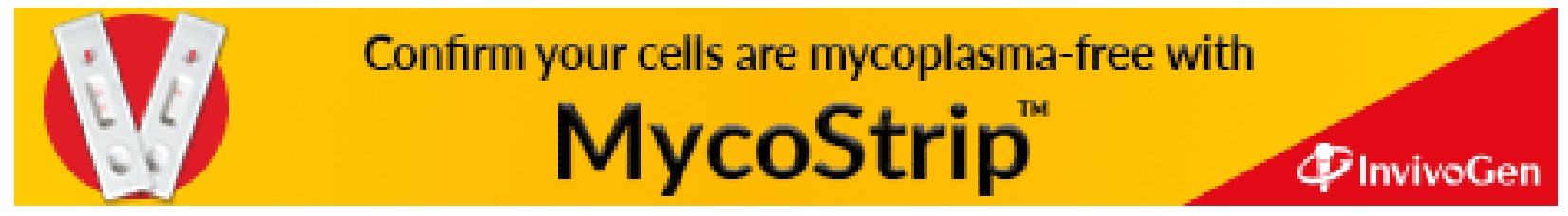

